American Journal of Applied Sciences 5 (11): 1474-1479, 2008

ISSN 1546-9239

(C) 2008 Science Publications

\title{
Characterization of Frequency Mode inside an Open Cavity for Different Shape Ratio
}

\author{
${ }^{1}$ Hallek Khouloud, ${ }^{1}$ Mourad Bouterra, ${ }^{1}$ Afif El Cafsi, ${ }^{1}$ Ali Belghith, \\ ${ }^{2}$ François Lusseyran and ${ }^{2}$ Pierre Gougat \\ ${ }^{1}$ Department of Physics, University of the Sciences of Tunis-Campus Academic 1060 Tunis Tunisia \\ ${ }^{2}$ LIMSI-CNRS Bat. 508 - B.P. 133-91 403 Orsay Cedex France
}

\begin{abstract}
A number of studies have referred to the existence of a vortex cell within an urban street canyon. The understanding of vortex dynamics or vorticity distribution is a such configuration is of great interest. This configuration was simulated by the interaction between a boundary layer and a cavity. Experimental characterization of the vortex structures evolution was developed by the technique of measure Particle Image Velocimetry (PIV). In this paper, we have studied and characterized experimentally by the technique of measure Laser Doppler Velocimetry (LDV) the vortex escape frequencies. Measurements were performed for different cavity shape ratio and for different mean velocities. Where the ratio between the shear instability process and the mechanisms of frequency selection which depend of the size of the cavity. The evolution of the system by the variation of the number of Strouhal according to the number of Reynolds, led us to a regrouping for every mode, permitting to really separate the mode the some of the other.
\end{abstract}

Key words: Cavity, vortex structures, frequency mode, spectral analysis, strouhal number, shape ratio

\section{INTRODUCTION}

In urban environment, the dynamic spatio-temporal of the fluid movements is there very complex, he occurred of movements of vortex, of very variable scale. The turbulence that occurred can have several reasons. Indeed, the presence of the obstacles, their geometry and their orientation are as many the factors that modify the dynamics and the level of the turbulence. The presence of buildings to the neighborhood of the urban setting, modify considerably the level of the turbulence and therefore the structure of the flow inside the street canyon. The dynamic comportment of the flow inside a cavity was the subject of a big number of experimental studies during the last forty years.

The vortex, as the wakes that developed inside a cavity, are the seat of instabilities and detachment phenomenon. These instabilities have consequences displayed on the structure of the flow; the amplification of the mixture plays a very important role in the thermal and mass transfer's phenomena. The possibility of monitoring this instability becomes a topic of nowadays a topic of actuality. Among the techniques utilized are those of active controls of the instabilities. Indeed, the formation of the vortex structure, emerging from the interaction between a boundary layer and a cavity, is broken down into two distinct phases; the first one corresponds to a process of amplification-convection of small disturbances in the flow initially existing near the cavity downstream corner, In fact, the mixing layer are developing above a cavity presenting a velocity profile having an inflection point which makes it unstable according to the hydrodynamic theory ${ }^{[1]}$. Consequently, within the mixing layer, the Kelvin-Helmholtz instability amplifies the initial disturbances which continue to grow in size until they form a large vortex structure that escapes towards the upstream which characterizes the vortex escape frequencies. he second phase is related to a retroaction to a distance generated by the interaction of the vortex with the upstream corner which has created new disturbances near the downstream corner. The exploitation of the display images in the latter zone highlights the vortex passage. These latter have been identified as it they were on a translation move defined by a velocity more or less equal to $\mathrm{U}_{\infty}{ }^{[2,3]}$. Kelvin-Helmholtz instabilities have been made visible in the cutting layer by the rolling up of the current lines. The big vortex structures that escapes toward the flow getting round the recirculation created by the upstream edge of the cavity ${ }^{[4]}$. The data processing by the technique of measure Laser Doppler

Corresponding Author: Khouloud Hallek, Department of Physics, $\quad$ University of $\quad$ Sciences, Tunis
Tunisia Tel: +21697622191 , Fax: +21671885073 


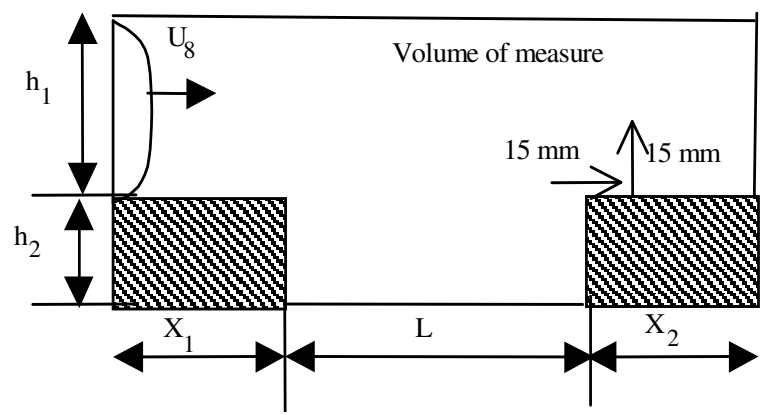

Fig. 1: Geometrical configuration

Table 1: Characteristic dimension of the cavities having constant $\mathrm{L}$

\begin{tabular}{llll}
\hline & Cavity $1 / 2 \mathrm{~h}_{2}$ & Cavity $1 \mathrm{~h}_{2}$ & Cavity $2 \mathrm{~h}_{2}$ \\
\hline $\mathrm{h}_{2}(\mathrm{~m})$ & 0.05 & 0.1 & 0.2 \\
$\mathrm{~L}(\mathrm{~m})$ & 0.1 & 0.1 & 0.1 \\
\hline
\end{tabular}

Table 2: Characteristic dimension of the cavities having constant $h$

\begin{tabular}{llll}
\hline & Cavity1 & Cavity3L4 & Cavity 1L2 \\
\hline $\mathrm{h}_{2}(\mathrm{~m})$ & 0.5 & 0.5 & 0.5 \\
$\mathrm{~L}(\mathrm{~m})$ & 0.1 & 0.75 & 0.5 \\
\hline
\end{tabular}

Velocimetry, in cavity upstream, has proved the existence of a well defined vortex escape frequency.

We are presenting in this paper the study of this frequency variation using the cavity upstream as well as its variation according to the cavity shape ratio. These results analysis reveals the existence of many modes within the flow which characterizes every cavity constant and by changing the length $\mathrm{L}$ of the cavity and keeping the height $\mathrm{h}_{2}$ constant.

\section{EXPERIMEN TAL DEVICE}

The experimental configuration is presented in Fig. 1 . The cavity characteristic dimensions are indicated in meters in Table 1 and 2 were $\mathrm{H}$ is the height, $\mathrm{L}$ the length and 1 the width of the cavity.

Velocity profiles in the boundary layer were obtained by hot wire measurements. The interaction region between the boundary layer and the cavity and the vortex burst from the cavity were characterized by Laser Doppler Velocimetry measurements.

Measurements were performed for different cavity shape ratio and for different mean velocity. The different shape ratio was obtained by changing the height of the cavity $h_{2}$ and keeping the length $L$.

\section{RESULTS}

To improve the characterization of the vortex structures were performed by PIV An example of visualization is presented in Fig. 2.

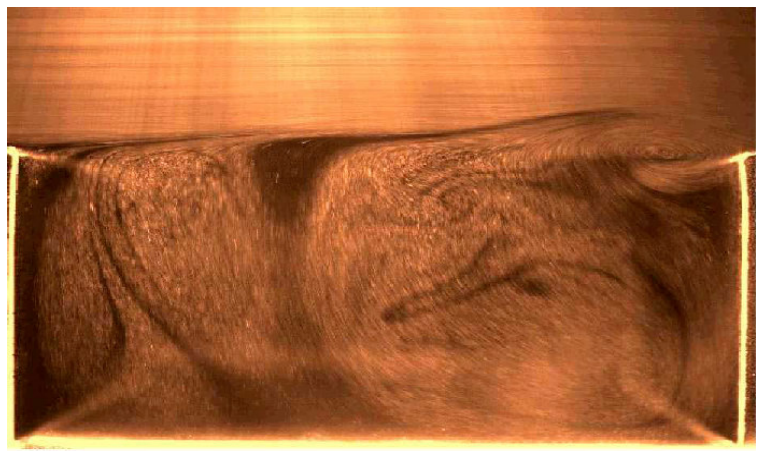

Fig. 2: Flow inside cavity, light sheet in the plane of symmetry

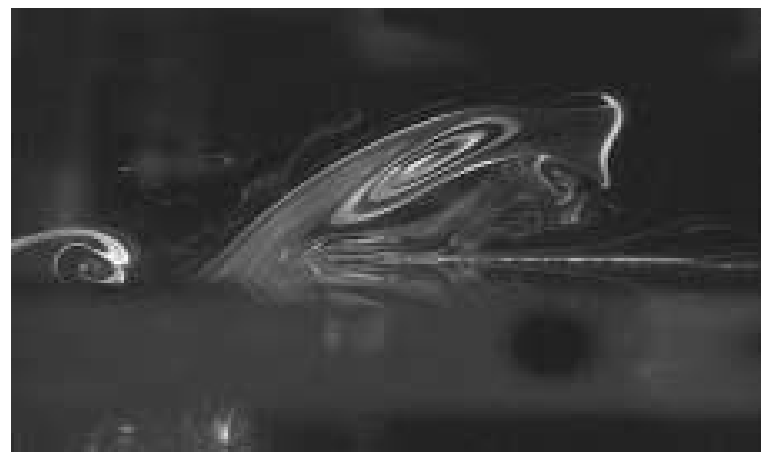

Fig. 3: Smoke visualizations of vortex release from the cavity $\left(\mathrm{h}_{2} / \mathrm{L}=1\right)$

Two counter rotation vortex are observed with eddies of smaller dimensions turning around them. The vortex has a diameter equal to the height of the cavity

Measurements make after the cavities showed that the boundary layer was no longer laminar. A spectral frequency analysis of the hot wire signals was realized which showed that the frequencies were in the flow frequency domain $(200 \mathrm{~Hz})$. This perturbation of the boundary layer was due to the exist of the vortex structures from the cavity. The flow visualization (Fig. 3) confirms the perturbation of the laminar boundary layer.

\section{VORTEX FREQUENCY FOR FIFFERENT CAVITIES SHAPE RATIO}

Measurements were performed for different cavity shape ratio for different mean velocities. The different shape ratio was obtained by changing the height of the cavity $h_{2}$ and keeping the length $L$ constant and by changing the length of the cavity and keeping the height $\mathrm{h}_{2}$ constant. The results of the vortex escape frequency corresponding to theses cases are presented in Fig. $4 \mathrm{a}$ 

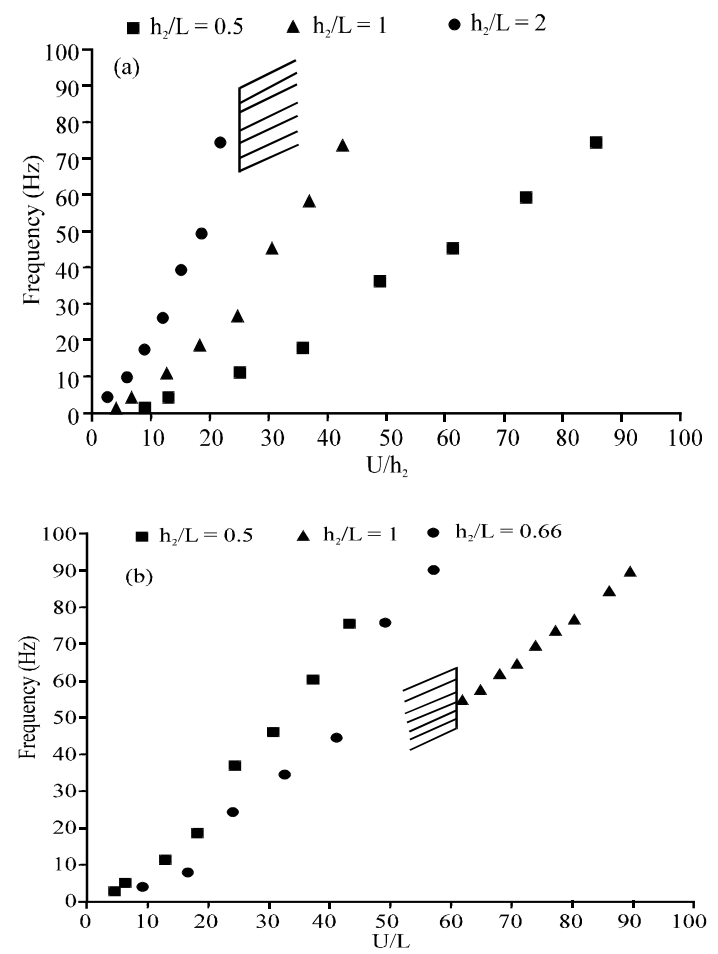

Fig. 4: (a) Vortex frequency as a function of $U / h_{2}$ for different cavities shape ratio $\mathrm{h}_{2} / \mathrm{L}$ ( $\mathrm{L}=$ constant), (b) Vortex frequency as a function of $\mathrm{U} / \mathrm{L}$ for different cavities shape ratio $\mathrm{h}_{2} / \mathrm{L}\left(\mathrm{h}_{2}=\right.$ constant $)$

and $b$ as a function of the adimensional factors $U / h_{2}$ and $\mathrm{U} / \mathrm{L}$ respectively. The vortex escape depends on the manner that the ratio was obtained, by changing the $h$ or the $\mathrm{L}$ cavity characteristic dimensions. For the shape ratio $\mathrm{h}_{2} / \mathrm{L}\left(\mathrm{h}_{2}=\right.$ constant $)$ and $\mathrm{h}_{2} / \mathrm{L}(\mathrm{L}=$ constant $)$ equal to 0.5 and for the same velocities there is no difference between the vortex escapes frequencies. We notice that for the shape ratio $h_{2} / L\left(h_{2}=\right.$ constant) equal to 1 there is no vortex escape for a mean velocity less than $3 \mathrm{~m} / \mathrm{s}$. $\mathrm{T}$ he frequencies performed in this case are sensibly greater then those obtained for the same shape factor $\mathrm{h}_{2} / \mathrm{L}(\mathrm{L}=$ constant $)$. For the case of a shape ratio $\mathrm{h}_{2} / \mathrm{L}$ ( $\mathrm{L}=$ constant) equal to 2 there is no vortex escape for a mean velocity more than $4 \mathrm{~m} / \mathrm{s}$. Measurements made for $\mathrm{h}_{2} / \mathrm{L}\left(\mathrm{h}_{2}=\right.$ constant $)$ equal to 2 showed that there is no vortex escape at all for any mean velocity value. Comparing the vortex escape frequencies for the same velocities for different $h_{2} / L$ ( $L=$ constant) value we notice that this shape factor has not a significant influence on the vortex escape behavior. On the contrary, analyzing the results obtained for the $h_{2} / L$ $\left(\mathrm{h}_{2}=\right.$ constant $)$ case we conclude that the length $\mathrm{L}$ of the cavity is a characteristic dimension which plays an important role in the vortex escape phenomenon In order to confirm this last result we have realized velocity measures in the escape zone for different cavities characterized by different ratios with the form $\mathrm{h}_{2} / \mathrm{L}$

Frequency variation according to the velocity with $h$ constant: The velocity measures in the detachment zone have realized for three cavities characterized with different ratio $h_{2} / L$; The different value of the shape ratio have obtained by keeping the height $h_{2}$ of the cavity constant $\left(\mathrm{h}_{2}=50 \mathrm{~mm}\right)$ and giving the length $\mathrm{L}$ of the cavity the values $100 \mathrm{~mm}, 750 \mathrm{~mm}$ and $50 \mathrm{~mm}$. The studied cavities will respectively be named cavity $1 \mathrm{~L}$, cavity 3L4 and cavity 1L2. On Fig. 5, we have drawn curves of the dominating frequencies variation according to the velocity for the three cavities. For each of these curves, we notice a linear increase of the frequency according to the velocity.

The adjustment of the curves by lines with the form $\mathrm{f}=\alpha \mathrm{U}_{\infty}+\mathrm{f}_{0}$ gives slopes with positive values, the results are presented on Table 3 . The intersection point of the adjustment line with horizontal axe of the velocities lets us define a critical velocity $U_{c}$ relating to the appearance of the observed profile. This critical velocity is no longer constant, which leads us to conclude that the length $\mathrm{L}$ is the main dimension which controls the vortex escape. This confirms that previous results ${ }^{[3,5,6]}$. From the critical velocity, we determine a critical Reynolds critique $\mathrm{R}_{\mathrm{ec}}$ for every observed mode.

\section{Variation of frequencies according to velocity for a large cavity (1L)}

Study of the large cavity $(\mathrm{L}=\mathbf{1 0 0} \mathrm{mm})$ : We are going to study in this paragraph, the evaluation of spectral density of energy, for velocity going from 0 to $5 \mathrm{~m} / \mathrm{s}$ and for cavity having shape ratio $h_{2} / L$ equal to $0.5\left(h_{2}=50\right.$ $\mathrm{mm}$ and $\mathrm{L}=100 \mathrm{~mm}$ ). Following the development of the frequency modes with an increasing flow rate, four main modes appear (Fig. 6).

The latest one (mode 3 ) corresponds to oscillation in a turbulent regime flow. The mode 2 has been identified with shear instability of the mixing layer above the cavity ${ }^{[2]}$. For $\mathrm{U}_{\infty}<1.4 \mathrm{~m} / \mathrm{s}$, one observes three modes (modes $0 ; 1$ and 2). For $U_{\infty}>1.4 \mathrm{~m} / \mathrm{s}$, the modes 0 and 1 disappear and the mode 2 persists until equal $U_{\infty}=2.3 \mathrm{~m} / \mathrm{s}$. The mode 3 intensifies quickly beyond a velocity of $2 \mathrm{~m} / \mathrm{s}$ and persists in the turbulent domain $^{[7]}$. 

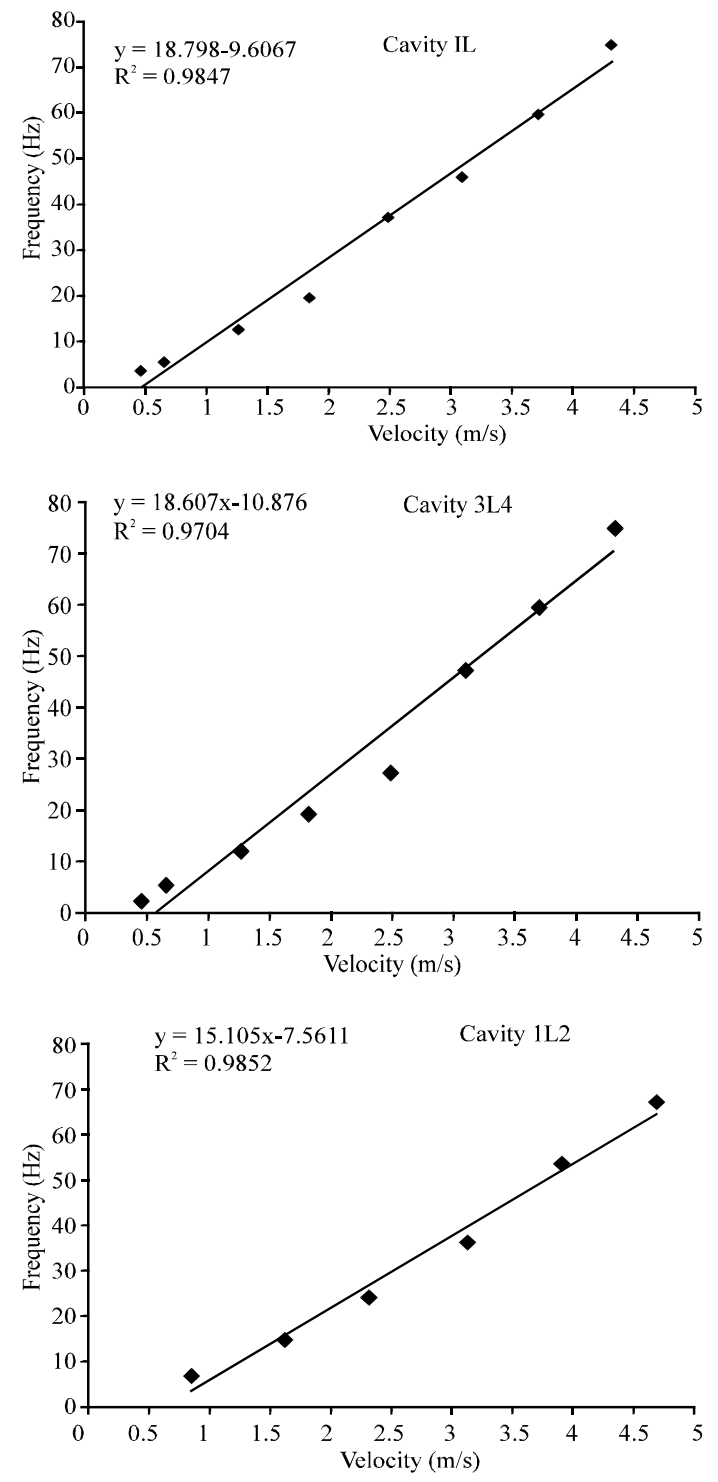

Fig. 5: Linear adjustment of the frequencies according to the velocity for the three cavities

Table 3: Parameter of the linear adjustment for the three cavities

\begin{tabular}{lllll}
\hline & $\alpha\left(\mathrm{m}^{-1}\right)$ & $\mathrm{f}_{0}(\mathrm{~Hz})$ & $\mathrm{U}_{\mathrm{C}}=-\frac{\mathrm{f}_{0}}{\alpha}$ & $\mathrm{R}_{\mathrm{ec}}=\frac{\mathrm{U}_{\mathrm{c}} \mathrm{L}}{\mathrm{v}}$ \\
\hline Cavity 1L & 18.798 & -9.606 & 0.51 & 3406 \\
Cavity 3L4 & 18.807 & -10.876 & 0.57 & 3853 \\
Cavity 1L2 & 15.105 & -7.561 & 0.5 & 3337 \\
\hline
\end{tabular}

The influence of shape ratio on instability modes: We are interested in this paragraph in studying and comparing the frequencies specter variations for velocity between $0.2 \mathrm{~m} / \mathrm{s}$ and $5 \mathrm{~m} / \mathrm{s}$ and for different form ratios of a cavity. These four ratios are obtained by keeping the height of the cavity constant

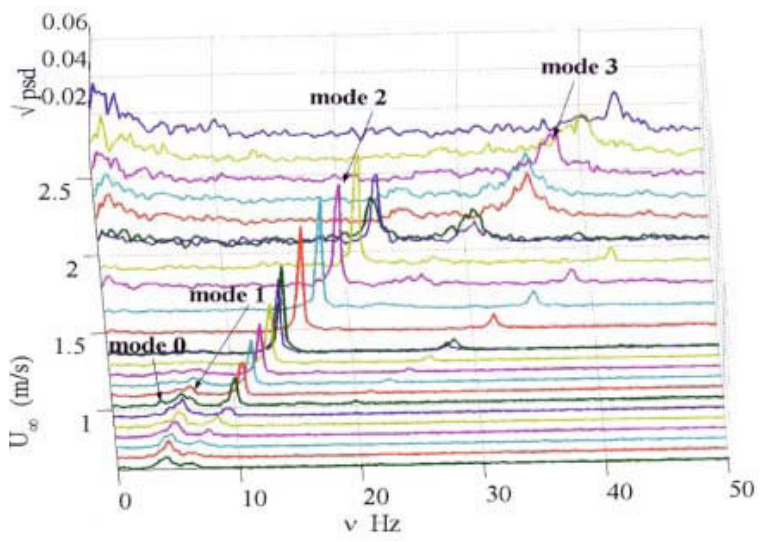

Fig. 6: Power spectral density of energy from horizontal velocity component by LDV upstream flow rate

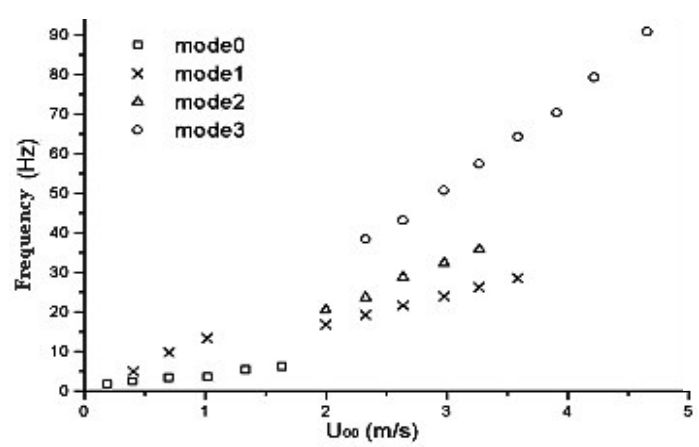

Fig. 7: Variation of frequency against $U_{\infty}$ and for every mode for the cavity $1 \mathrm{~L}$

$\left(\mathrm{h}_{2}=50 \mathrm{~mm}\right)$ and giving to the length $\mathrm{L}$ the value $100 \mathrm{~mm}, 75 \mathrm{~mm}$ and $50 \mathrm{~mm}$. The way of proceeding lets us analyze the effect of the cavity length variation on the frequencies specters. The studied cavities will be named respectively 1L, 3L4 and 1L2. This study have been realized from the recording of $150 \mathrm{~s}$ temporal series obtained by LDV, longitudinal component of the velocity, in a point situated $15 \mathrm{~mm}$ from the cavity edge and $15 \mathrm{~mm}$ from the wall (Fig. 1).

Results relative to the cavity 1L: The analyses of the frequencies specters relative to the cavity $1 \mathrm{~L}$, shows the existence of four modes. On Fig. 7, we have presented the evolution of the frequencies according to the velocity for every mode, for each of these curves, we have recorded a linear growth of the observed frequencies. The curves adjustment by the lines gives the some of the results presented on Table 4. Primary analysis of the given results in this table, shows that the velocity critical of the mode 2 is twice as near as velocity critical of the mode 0 and that the velocity 
Am. J. Applied Sci., 5 (11): 1474-1479, 2008

Table 4: Results of linear adjustment for the cavity1L

\begin{tabular}{lllll}
\hline & $\alpha\left(\mathrm{m}^{-1}\right)$ & $\mathrm{f}_{0}(\mathrm{~Hz})$ & $\mathrm{U}_{\mathrm{C}}=-\frac{\mathrm{f}_{0}}{\alpha}$ & $\mathrm{R}_{\mathrm{ec}}=\frac{\mathrm{U}_{\mathrm{c}} \mathrm{L}}{\mathrm{v}}$ \\
\hline Mode 0 & 6.56 & -0.75 & 0.114 & 762 \\
Mode1 & 7.41 & 1.91 & - & - \\
Mode 2 & 12.13 & -2.53 & 0.208 & 1391 \\
Mode 3 & 17.07 & -5.61 & 0.328 & 2192 \\
\hline
\end{tabular}

Table 5: Results of linear adjustment for the cavity 3L4

\begin{tabular}{lllll}
\hline & $\alpha\left(\mathrm{m}^{-1}\right)$ & $\mathrm{f}_{0}(\mathrm{~Hz})$ & $\mathrm{U}_{\mathrm{C}}=-\frac{\mathrm{f}_{0}}{\alpha}$ & $\mathrm{R}_{\mathrm{ec}}=\frac{\mathrm{U}_{\mathrm{c}} \mathrm{L}}{\mathrm{v}}$ \\
\hline Mode 0 & 3.043 & 1.035 & - & - \\
Mode 1 & 6.662 & 4.229 & - & - \\
Mode 2 & 12.436 & -15.785 & 0.379 & 2526 \\
Mode 3 & 22.478 & -5.61 & 0.702 & 4680 \\
\hline
\end{tabular}

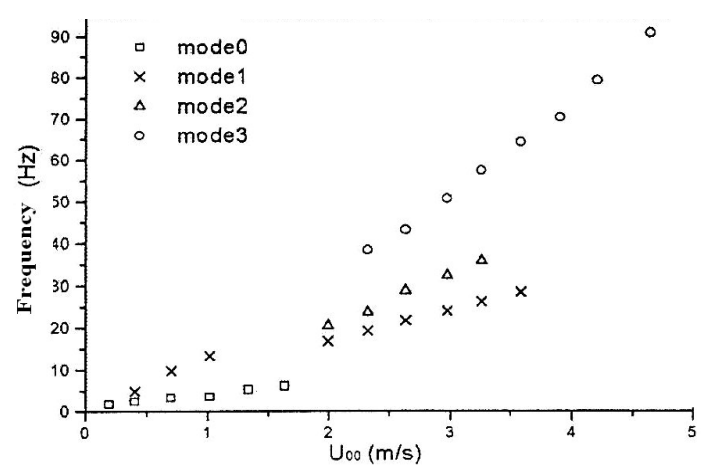

Fig. 8: Variation of frequency against $\mathrm{U}_{\infty}$ and for every mode for the cavity $3 \mathrm{~L} 4$

critical of the mode 3 is the triple of the velocity critical of the mode 0 . Notwithstanding, the recorded frequencies for mode 1 does not present a critical velocity and seems to present another oscillatory phenomenon the critical velocity.

Results relative to the cavity 3L4: On Fig. 8, we present the frequencies variation according to the velocity obtained from the cavity 3L4. The general appearance of these curves is similar to those obtained by the cavity $1 \mathrm{~L}$. The results are on Table 5 . We notice that the mode 1 does not always present critical velocity and that the coefficient and of the adjustment of the frequencies curves of this mode have the same orders as the determined coefficient for the cavity1L. This leads us to believe that the mode 1 independent of the cavity length so it is independent of the form ratio. We note that obtained critical velocity for the mode 2 is near to that obtained for the mode 3 .

Results relative to the cavity 1L2: On Fig. 9, we present the results from the cavity $1 \mathrm{~L} 2$ who is characterized by four modes. The four modes obtained have got a positive slope. On Table 6 , the critical
Table 6: Results of linear adjustment for the cavity 1L2

\begin{tabular}{lllll}
\hline & $\alpha\left(\mathrm{m}^{-1}\right)$ & $\mathrm{f}_{0}(\mathrm{~Hz})$ & $\mathrm{U}_{\mathrm{C}}=-\frac{\mathrm{f}_{0}}{\alpha}$ & $\mathrm{R}_{\mathrm{cc}}=\frac{\mathrm{U}_{\mathrm{L}}}{\mathrm{v}}$ \\
\hline Mode 0 & 1.6589 & 1.7507 & - & - \\
Mode 1 & 2.7451 & -4.0306 & 1.468 & 9788 \\
Mode 2 & 6.5513 & 4.2649 & - & - \\
Mode 3 & $810^{-4}$ & $1410^{-4}$ & - & - \\
\hline
\end{tabular}

Table 7 : Valeurs de $\mathrm{St}_{\infty}$ obtenues pour différentes cavités

\begin{tabular}{llll}
\hline & $\mathrm{St}_{\infty}$ Cavity $1 \mathrm{~L}$ & $\mathrm{St}_{\infty}$ Cavity $3 \mathrm{~L} 4$ & $\mathrm{St}_{\infty}$ Cavity 1L2 \\
\hline Mode 1 & 0.656 & 0.48 & - \\
Mode 2 & 1.213 & 1.096 & 0.621 \\
Mode 3 & 1.707 & 1.49 & 1.123 \\
\hline
\end{tabular}

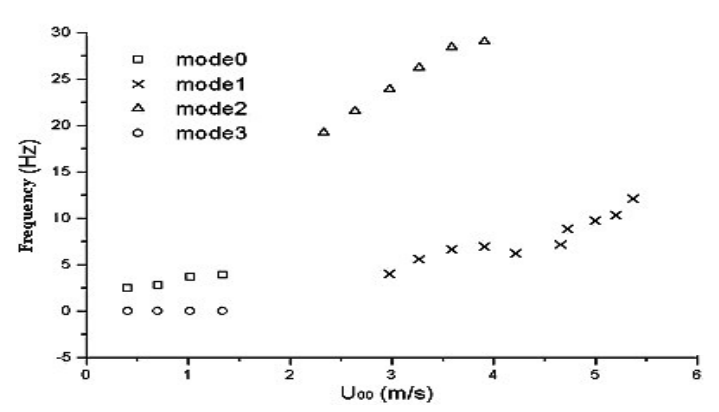

Fig. 9: Variation of frequency against $\mathrm{U}_{\infty}$ and for every mode for the cavity $1 \mathrm{~L} 2$

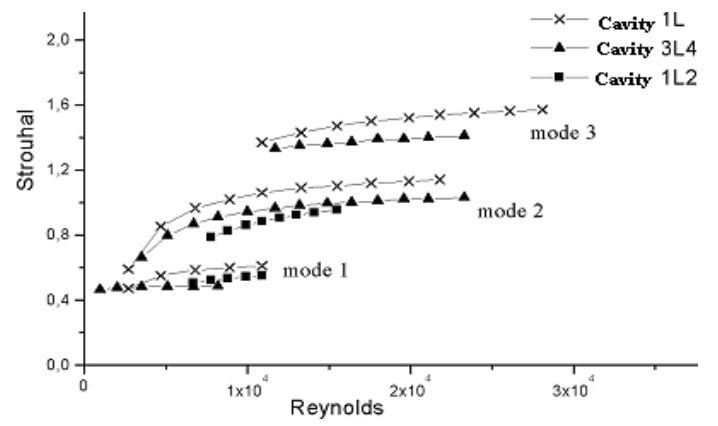

Fig. 10: Variation of Strouhal against Reynolds: 3 modes grouping effect

velocity relative to this cavity, are only obtained for the modes 2 and 3 . The critical velocity of mode 3 is not far from double of the critical velocity of mode 2 .

The different values of $\mathrm{St}_{\square}$ gotten for the different cavities and the different modes observed are regrouped in the Table 7. On Fig. 10, we represented all modes observed for the different cavities. We notice, for a number of Reynolds superior to $10^{4}$, that the analysis of these results driven well to a regrouping for every mode, permitting to really separate the mode the some of the other ${ }^{[8]}$. We also note that for a same cavity, the number of Strouhal increases 0.5 on average at the time of the passage of one mode to the mode following. 


\section{CONCLUSION}

The study presented in this paper has allowed us to emphasis some characteristics of the vortex structures origination the interaction between boundary layer and a cavity. The experimental measures have been realized by (LDV). The recordings analysis of the temporal series of the component longitudinal of the upstream velocity of the cavity reveals the existence of many modes within the flow. This study shows that the appearance of the frequencies modes depends on the cavity and his shape ratios. The study of the frequencies specters according to the velocity reveals the existence of the critical velocity, the appearance characteristics of every frequency mode. The evolution of the system by the variation of the number of Strouhal according to the number of Reynolds, led us to a regrouping for every mode, permitting to really separate the mode the some of the other.

\section{ACKNOWLEDGEMENTS}

We would like to acknowledge LIMSI-CNRS, France for providing the Short-Term Grant to support this project.

\section{REFERENCES}

1. Chatellier, L., J. Laumonier and Y. Gervais, 2006. Active control of the aeroacoustics of cavity flows from the downstream edge, C. R. Mecanique 334259-265
2. Elcafsi, A., A. Rambert and P. Gougat, 1999. Optical flow technique used to characterize the flow inside different cavities interacting with a boundary layer. Euromech 406 Image Processing Methods in Applied Mechanics, Warsaw, Poland.

3. Rambert, A., A. Elcafsi and P. Gougat, 2000. Entrained cavity. Lecture Notes in Physics. Vortex structure and dynamics. pp: 283-290, Springer.

4. Elcafsi, A., 2003. Caractérisation expérimentale et étude fréquentielle des détachements tourbillonnaires issus de l'interaction couche limite-cavité. Thèse principale.

5. Bouchet, M.G., 1996. Etude expérimentale et numérique des auto-oscillations d'un jet confiné. Thèse de doctorat, Université Paris VI.

6. Maurel, A., P. Ern, B.J.A. Zielinska and J.E. Wesfreld, 1996. Experimental study of selfsustained oscillations in a confined jet. Physical Review E, Vol. 54, n 4, pp: 3643-3651.

7. Lusseyran, F., P. Gougat, A. Rambert, Y. Fraigneau and A. Elcafsi, 2002. Modes spatiaux et modes fréquentiels dans une cavité en interaction avec une couche limite: Confrontation calculexpérience à nombre de Reynolds modéré. $8^{\text {ème }}$ Congrès Francophone de vélocimétrie laser, Orsay.

8. Lusseyran F., P. Gougat, Y. Fraigneau and A. Elcafsi, 2003. Caractéristiques spatiales et temporelles de modes instables en cavité ouverte. $16^{\text {ème }}$ French colloque of mechanics, Nice, France. 